\title{
Xpertum: Competences and Social Networking
}

\author{
José Arturo Tejeda Gómez, Agustí Sancho Martínez, Esteve Allmirall \\ UPC - Universitat Politécnica de Catalunya, Jordi Girona 1-3 Campus Nord-Building Omega, 08034 \\ Barcelona, Spain, \{jatejeda, asancho, almirall\}@lsi.upc.edu
}

\begin{abstract}
We present Xpertum, a web-based tool for visualizing social networks to provide valued insights in the knowledge and innovation management processes. Our approach is based on networking between individuals and competences. Since people could gain experience in several knowledge areas, we consider the competences as levels of knowledge in a certain technical area or discipline, which users are dynamically involved inside the organizations. Large communities of individuals offer a significant complexity not only for visualization but also for understanding and explore. We suggest algorithms to highlight the relationships between both entities, individuals and competences, which are being visualized. The tool deals also with clustering of entities and work team assembly.
\end{abstract}

\section{Keywords}

Visualizations, Social Networks, Competences

\section{Introduction}

The innovation process is used to describe basically an array of sources and objectives that culminate in the act of innovation [Gellatly, Peters, 2000]. The innovation takes place inside of knowledge cycle (acquisition, assimilation and development) [Ho 2007]where it leads to creating or discovering new knowledge or technology, thus a new value could be created by applying the new knowledge to actual business or social challenges. There is no doubt that innovation is market-driven since it depends on its commercial value.

The knowledge can be divided into two categories: tacit and explicit, the first one can be easily expressed in words and numbers; the second one is context-specific, highly personal and hard to formalize and includes, among other things, intuitions and experiences [Nonaka 1998]. Nowadays, the organizations deal with metrics that capture the level of knowledge that its employees have in a certain moment, including both types of knowledge. Based on it, the leaders can take decisions to assemble teams, hire new employees or simply be aware of its technical potential.

The innovation requires multidisciplinary perspectives to get a high commercial value. Innovation needs a balanced intellectual attitude and level of knowledge as well, in order to find all problems-solutions. The level of knowledge can be represented as a competence. Even though, the concept of competence has different meanings, we have focused on the following: a competence is defined as level of knowledge based on the results of previous actions to enrich the know-how [Ryle 1949] in certain technical data or discipline. That being so, a set of competences could be found among people involved in the innovation tasks.

We take in account that highlighting the potential of networking employees could help to increase productivity and speed of innovation in companies [Cohen, Prusak, 2001]. A social network analysis maps the relationships between people or groups to understand how these relationships could help or being an obstacle to the knowledge flow and human interaction.

In this paper, we describe the social network representation, architecture and visualizations that xpertum deploys. In our field trial, we explore the algorithms that could represent the social networks and the relationships among individuals and competences, in order to provide an 
aesthetically visualization. We follow with the work in progress to implement the work team assembly and profile recommendation. Finally, we conclude with discussion of lessons learned.

\section{Social networks and software}

Social network theory represents social relationships in terms graphs (nodes and edges) [Freeman 2000]. Nodes represent individuals and edges are the relationships between them. If all nodes in the social network are categorized in the same type, the network is a one-mode network, otherwise is an n-mode network [Asratian et al. 1998].

In social networks represented as graphs, it could find edge and weighted-edge, the first one only represents a relationship between nodes, the second one represents the strength of relationship between the nodes in a measured way. The type of weights will depend on how the social network is built and the kind of relation that it represents.

Visualization of social networks has provided new insights to researchers about structure and shape about it. There are several tools to visualize social networks to visualize social networks and its possible links with concepts (such as: tags). However, most of these tools could be complex to manipulate (several visualization with non-precise meaning), support visualizations of their own data or access-restricted. Moreover, the majority of visualization provided by tools are egocentric (focused on individuals) and it is not useful when relationships between concepts and users have to be highlighted as well.

There is an application-oriented social networking perspective that defines certain functionalities, which have been proposed as Social Networking Services (SNS) [Richter, Koch 2008], the basic functionalities are: Identity management, expert finding, context awareness, contact management, network awareness and exchange.

In innovation process, the interactions between involved people are fundamental aspect to produce innovative solutions or ideas. Even though, that process could be complex, it is necessary to provide the SNS functionalities to assure a productive innovation process.

Our approach to fostering innovation, within the frame of Laboranova, is based on augmenting social capacities of innovators, i.e. enhance their social networking and their sharing of concepts and ideas [Andrade, et al. 2007].

This augmentation of the social capacities is achieved by focusing in three main aspects:

- Visualization of the relations between people, concepts and people-concepts. This aspect provides a clear picture of the overall distribution of knowledge among the organization and the individuals.

- Selection and recommendation of new concepts / ideas to people. Since there are large organizations, the amount of knowledge that they can retain is huge. Having such a big knowledge base requires some mechanisms to allow innovators to explore and to reach the knowledge they are more interested in.

- Diffusion of knowledge in the community. The knowledge that is just stored in databases does not create more value, so innovators should spread ideas to other innovators to collaborate more actively in the development of those ideas.

We are aware of these described needs to develop Xpertum, basically we have addressed these needs in the following requirements: general purpose visualizations (tackling different scenarios as: concepts-individuals, concepts-concepts and individuals-individuals), provide selections and recommendation mechanisms to assemble a work team and an interface to be accessed by any organization to visualize its own data in the tool. In the next section, we will detail all of them deeply. 


\section{Xpertum}

The overall approach of Xpertum is to provide an interactive web-based tool that supports a well-defined set of social network visualizations and team assembly, for foresting the innovation process. We decided to develop a web-based tool in order to be accessed by any organization or electronic site, thus we do not restrict the access. The social network visualizations have been defined to provide a useful view about aspects as: relationships between individuals-toindividuals, individuals -to-concepts, concepts-to-concepts. We have concretized the concept entities in competences, according to [Ryle 1949], to facilitate the visual analysis of the social environment (people connected with competences) or work team assembly. The main objective to connect individuals and competences is to highlight the relationship between them through the level of knowledge from individual to certain competence(s).

In terms of SNS, Xpertum concerns the following functionalities:

- Identity management: it only provides user identity information such as: email, telephone, etc., allowing contact establishment among all users in the network. The availability of users identity information have to be managed by the own organization.

- Expert search: it supports this functionality in two ways: implicit (visualizations) and explicit (team assembly). The last one is still work in progress.

- Context Awareness: the user can realize about common contacts and common knowledge areas in his/her own social environment through visualizations.

The types of visualizations provided in Xpertum are: graphs and treemaps. Since graph is the common representation of social networks and it is easy to highlight the relationships between our entities: individuals and competences. And, the treemap produce a visual representation of quantitative features of a clustering model.

The selected algorithms have the main objective to present an aesthetical visualization to user. We have selected geometric, force and clustering layout to present different kind of visualizations. All the algorithms and layouts will be described in the next sections.

Xpertum has been developed to load data from GraphML file, which can be stored in any webserver or uploaded to xpertum server. The total number of nodes that supports xpertum is 500 (competences plus individuals).

The architecture of xpertum is basically server-client, all the visualization operations take place on the xpertum server and the graphic presentation on the client machine.

Since, work team assembly is a work in progress but we will describe the main objective of this functionality in section 3.4 .

\subsection{Competences and individuals representations}

Measuring the level of knowledge, such as competence, it is not an easy task, the issue of measuring remains as challenge of knowledge management. At this point, we assumed that users can be evaluated by themselves or by others and we just only manipulate the value of the given rating.

A two-level hierarchical structure of competences have been defined to classify competences in areas of knowledge, in such a way, we group competences in several areas to provide a compact way to visualize all the competences. For example, it could classify computing area in computer architecture, software design, etc and electronic area in devices, circuits, etc.

The number of competences and individuals have to be provided by the organization that is interested in deploy Xpertum. In that way, the organization protects and filters the data to be loaded by the tool. 
In order to visualize individuals and competences, the following information included in the GraphML file is required:

- User Nodes (user profile)

- Competence Nodes (name and area of knowledge)

- Edges (relationships between users and competences with a given value)

The edges have to be rated between 0 and 1, otherwise this value has to be mapped to that range; where zero means "not capable" on and one "is totally capable". The number of relationships is also important to present the graphic element into visualization. While more relationship has a node, the attached halo of the node will grow up. In the same way, the color is important as well, we have differentiated between individuals and competences. Individuals will be showed in gray color and competences will be showed in different colours but gray.

\subsection{Visualizations}

The following types of visualization have been implemented in xpertum: global, individual and competences. All of them count with the following layouts: galaxy, big bang, treemap and clustering views. The users can change the elements types that are being visualized. They could try only individuals, only competences or both at the same time, depends on the type of visualization.

\subsubsection{Global, Individual and Competences Views}

The Global View offer an entire view of the social network that includes all competences from areas and individuals connected with (c.f. figure 1)..

The Individual View only presents the individual nodes and their relationships based on which competences are connected between them. It can

Finally Competences View only presents the competences nodes and their relationships based on which individuals are connected between them.

\subsubsection{Galaxy View}

We implemented a customized algorithm to display the global view based on distances between the competences which are related to some individual.

Basically, the algorithm displays the graph of persons and competences in 2 stages. The first stage orders the affiliation nodes in an ellipse around the centre of the display area. The second one orders the nodes of individuals into the area of ellipse. The position of each individual was computed in function of the position of their competences and the value (strength) of the relationships between each pair individual-competence. A Bipartite graph is applied to this visualization, since it shows and connects both kinds of nodes [Asratian 1998].

\subsubsection{Big Bang Layout}

Another approach to visualize graphs is the force-directed and energy-based methods for creating straight-line drawings of undirected graphs [Noack 2003]. In force-directed algorithms, the model is a force system then the algorithm tries to find equilibrium where the total force on each node is zero. The energy algorithms, uses an energy model and algorithm that searches a state with minimum total energy.

The Kamada-Kawai Algorithm is the algorithm that provides this kind of visualization. Since the distance between nodes in the space is determined by their graph-theoretic distances, it produces a spread mesh of nodes where it is clear the position of each node. We compare with Fructerman-Reingold and Spring Layouts but Karamada-Kawai keeps a well-distributed nodes. 


\subsubsection{Clustering Layout}

The clustering techniques in graphs are applied to obtain groups of the node which are connected among them and to obtain visualizations that display the well-distributed and well-separated clusters.

We found two main clustering algorithms to be applied for Xpertum: LinLog (LL) [Noak 2003] and Markov Cluster Algorithm (MCL) [Van Dogen 2008]. The first one is energy-based cluster algorithm and the second one is based on simulations of flow in graphs. We selected LinLog since it produces a better visualization and it groups the nodes in a well-separated way, the opposite to MCL and it has not a suitable configuration input values when the size of the graph grows up.

The obtained visualization by LL is an unconnected graph and the relationships between the elements are showed by distance proximity (c.f. figure 1).

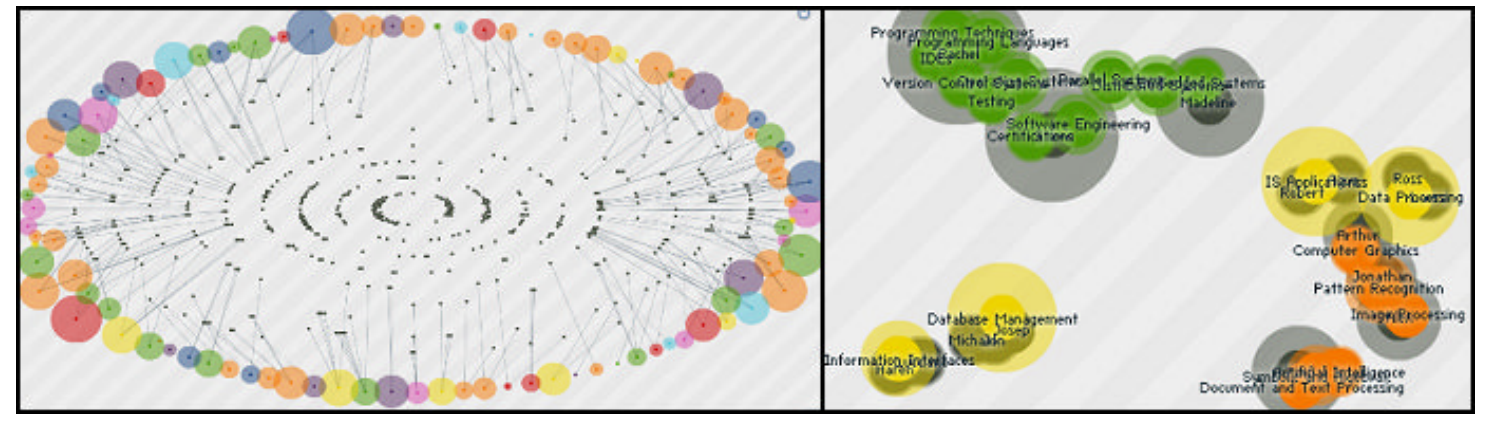

Figure 1. Global View and Galaxy and Clustering sub-views applied.

The visualization graph algorithms consider the nodes like a point, without thickness, without shape and in our circle representation, without radius.

All the presented visualizations, despite its mathematical precision, might not be visually understandable; therefore we decided to apply collision avoiding techniques to solve the problem.

With this goal we developed a collision algorithm that subtly modifies the positions nodes without change the general visualization shape. This algorithm is implemented in the visualization layer to ensure that the domain is not affected and the different clients can choose how to process the common information that the domain of Xpertum provides.

\subsubsection{Treemap Layout}

The treemap algorithm partitions the display space into a collection of rectangles, forming a mosaic. Each rectangle pertains to one type of node and is sized (the physical area the box takes in the display space) according to a quantitative value related to number of connections with other type of node, for example: if we want to present a treemap related to individuals, each rectangle represents the number of competences connected with and viceversa. We selected this layout taking account the following characteristics of this kind of visualization [Bruls 1999]:

- It offers a visual representation of quantitative features of a clustering model, show the hierarchical aspects of a clustering model

- It provides an interactive exploration of the hierarchy of a clustering model

- It makes full use of available display space

- It is not affected by the size of the underlying network.

On their own, these characteristics are not unique, but a treemap uniquely combines all of these benefits into a single tool, thus its attractiveness to social network analyst. 
In our project, we apply the squarified treemaps technique over competences and individuals using 2 steps, the areas and the nodes.

At the first step, we apply the treemap algorithm to areas to show how relevant is the group of individuals/competences that are included.

For the second step, for each treemap element that we obtained at the first step, we apply the treemap algorithm for each element to show how relevant an competence or a individual could it be.

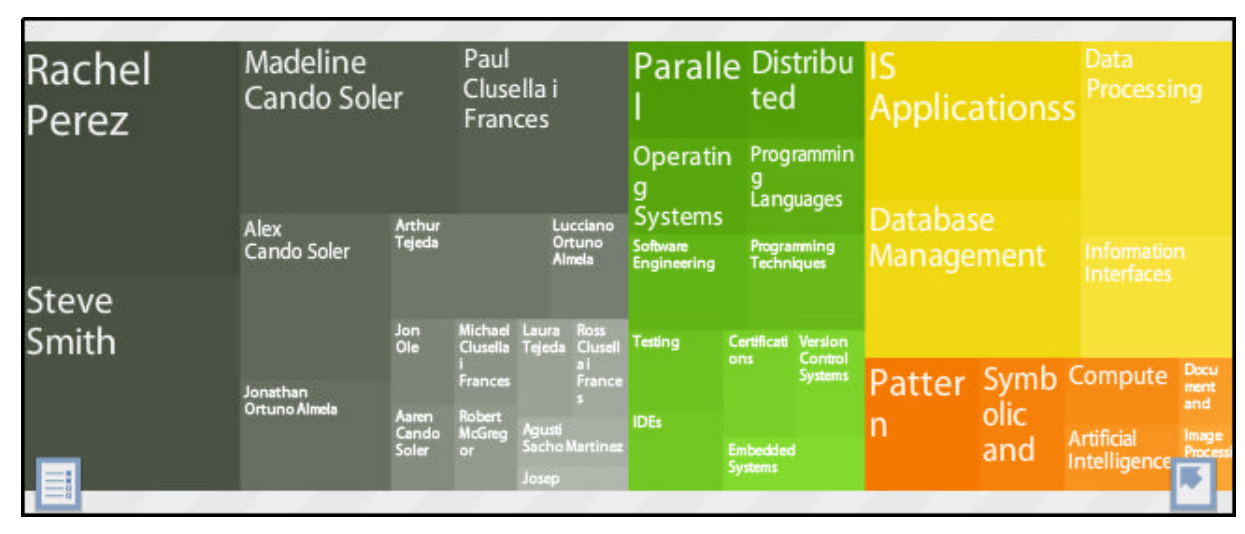

Figure 2. Treemap layout in Global view.

\subsection{Architecture}

Xpertum have been developed with three-layer architecture (cf. Figure 3). The domain layer is responsible of the data processing from data sources that can be independent of the application and provides the required visualization. This was currently being performed entirely by a flash application, but thanks to the new architecture, the series of data sources that can provide the required data for Xpertum is unlimited, as is the environment or the devices that can act as the client of the visualization, ranging from flash widgets to iPhone apps.

The main advantage of this architecture is the versatility and transparency of data processing while increasing the performance and resource efficiency in the user side. Another collateral advantage is that building Xpertum in separate modules offers the development team to increase the number of algorithms and visualizations available transparently to the user.

Finally, to obtain data from any external databases, we added to the architecture a new component, the data interface, that is the responsible of translates the external data to a XML format that the Data Manager of Xpertum can understand.

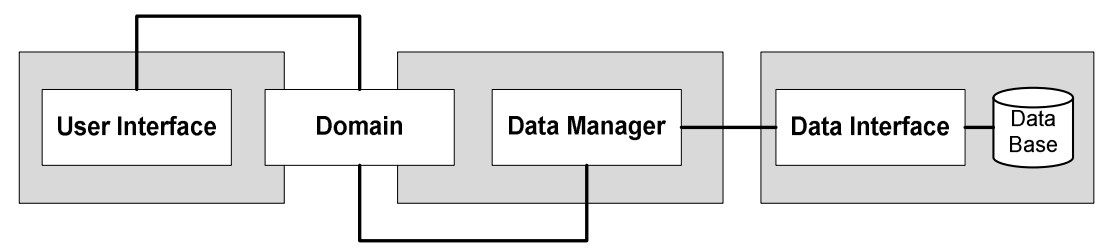

Figure 3. Xpertum Architecture

\subsubsection{User Interface}

This layer provides the user interactions with the applications and is responsible for displaying the visualizations to the users. The requests of new petitions of visualizations are sent by this layer to the domain. 


\subsubsection{Domain}

This layer receives the requests of User Interface layer and establishes an interaction with the data manager when visualization is required. The Graph visualizations are computed in Xpertum server and the treemap visualizations, that not require so much computational cost, are computed in Xpertum Mashup.

\subsubsection{Data Manager}

This layer is the responsible of manage the data and obtains the data from external sources, (such as: files or Data Interfaces) and send it to domain when it is required.

\subsubsection{Data Interface}

It is responsible for translate the database content to a GraphML format. We decided to use an specific formatted filed base on XML, named GraphML. Since XML (Extensible Markup Language) is a standard extensible meta-language developed by World Wide Web Consortium (W3C) and provides semantic for the human mind to work with graph data without the help any programs.

\subsection{Work Team Assembly}

We have found two main lines to analyze a work team assembly: analysing the interaction among the members of the network. On one hand, in order to set a work team, it has been proposed the use of several types of links between individuals, each link represents the type of individual such as: rookies, experts, etc. then distribution of different types of edges or connections reflects the diversity of the team. For example, if teams have more expert links, representing interactions between expert individuals, it is less likely that they will have innovative ideas because their shared experiences tend to homogenize their pool of knowledge. In contrast, teams with a variety of types of links are likely to have more diverse perspectives to draw from and therefore to contribute more innovative solutions [Guimerà 2005].The collaboration between people involved in the innovation process have been also tackled in [Guimerà et al. 2005], the main outcome suggest that there are optimal values for diversity and level of expertise in the work team that encourage and produce innovative solutions.

On the other hand, there are several metrics for analyzing a social network such as: density and centrality. The first one, density, describes the overall level of interaction between the members of the network. Centralization reflects the extent to which interactions are concentrated in a small number of individuals. [Sparrowe 2001].

We are focusing on assemble a work team by finding users on the network that could satisfy certain input values related to a set of competences. Basically, the system will select the suitable users to assemble a team with the given set of competences and respective values.

\section{Conclusions and future work}

We presented a competences and social networking tool which is not only limited to provide visualizations but it adapts to social environment where knowledge concepts could be measured with a concrete value. Through xpertum, team leaders, human resources departments and others persons involved into innovation process could deploy this tool to analyze their social environment and/or to assemble a work team.

Our future work will focus on adding egocentric visualizations and automated (applying diversity theory) mechanisms to assemble a work team, including profile recommendation. We will also include analysis and simulations about social network environments by mixing both perspectives tackled in [Guimerà 2005] and [Sparrowe 2001], to establish a possible relationship between density and centrality measure among different kind of relationships into work team. 


\section{Acknowledgement}

This work has been partly funded by the European Commission through IST Project Laboranova: Collaboration Environment for Strategic innovation (No. IST-5-035262-IP) of the $6^{\text {th }}$ Framework Research Programme. The authors wish to acknowledge the Commission for their support. We also wish to acknowledge our gratitude and appreciation to all the Laboranova project partners for their contribution during the development of various ideas and concepts presented in this paper. Finally, our acknowledge to Knowledge Engineering and Machine Learning Group (KEMLg) members for their support as well.

\section{References}

Andrade, Z; Tejeda, A; Almirall, E (2007) Application-oriented research on tools for augmenting social capacities. Laboranova, Deliverable D4.2.1, 2007.

Asratian, A. S; Denley, T. M. J; Häggkvist, R. (1998) Bipartite Graphs and their applications. Cambridge Univerisity Press, 1998.

Bruls, M. et. al. Squarified Treemaps (1999). In: W. de Leeuw, R. van Liere (eds.), Data Visualization 2000, Proceedings of the joint Eurographics and IEEE TCVG Symposium on Visualization, 2000, Springer, Vienna, p. 33-42.

Cohen, D.; Prusak, L. (2001) In Good Company: How Social Capital makes Organizations Work, Harvard Business School Press, Boston, 2001.

Freeman, L. C. (2000) Visualizing Social Networks. Journal of Social Structure. Vol 1.

Gellatly, Guy; Peters Valerie. (2000) Understanding the innovation process: Innovation in Dynamic Service Industries. Analytical Studies Branch, Research Paper Series. Statistics Canada, Micro-Economic Analysis Division, No 11F0019MPE, No 127.

Guimerà,R; Uzzi, B; Spiro, J; Nunes, L.A. (2005) Team assembly mechanisms determine collaboration network structure and team performance, Science 308, 2005, p. 697-702.

Ho, D. (2007) Research, Innovation and Knowledge Management: the ICT Factor. UNESCO, Commissioned Paper Series.

Laboranova: Collaboration Environment for strategic innovation. WWW page. http://www.laboranova.com/index.html, accessed 22.5.2009.

Noack, Andreas. (2003) An Energy Model for Visual Graph Clustering. Proceedings of the 11th International Symposium on Graph Drawing, 2003, LNCS 2912.

Nonaka, I; Konno, N. (1998) The Concept of "BA" Building a Foundations for Knowledge Creation. p.53-67. Knowledge Management, Critical Perspectives on Business and Management. (Ed) Ijukiro Nonaka. Routledgefalmer, 2005.

Richter, A; Koch M. (2008) Functions of Social Networking Services. COOP'08. International Conference on the Design of Cooperative Systems.

Ryle, G. (1949) The Concept of Mind. In: Gracia, J; Reichberg, G. M; Schumacher, B.N. (Eds): The Classics of Western Philosophy, a Reader's Guide. Blackwell Publishing, 2003.

Sparrowe, R. T; Liden, R.C; Wayne, S.J; Kraimer, M. L. (2001). Social networks and the performance of individuals and groups. Academy of Management Journal 2001

Van Dongen, S. (2008) Graph clustering via a discrete uncoupling process. Siam Journal on Matrix Analysis and Applications Vol 30 Issue 1, p. 121-141.

Viégas, F; Donath J. (2004) Social Network Visualization: Can We Go Beyond the Graph?. Workshop on Social Networks. CSW’04, Chicago, IL, USA, 2004. 